\title{
A New Class of Supersymmetric Orientifolds with D-branes at Angles
}

\author{
Ralph Blumenhagen; Lars Görlich ${ }^{\dagger}$ and Boris Körs ${ }^{\ddagger}$ \\ Humboldt Universität zu Berlin, Institut für Physik, Invalidenstr. 110, 10115 Berlin, Germany
}

\begin{abstract}
We describe a new class of supersymmetric orientifolds which combine the world-sheet parity transformation $\Omega$ with a complex conjugation in the compact directions. As an example, we investigate in detail the orientifold of the $\mathbb{Z}_{3}$ toroidal orbifold in six and four dimensions. We demonstrate how the solution to the tadpole cancellation conditions, the resulting gauge groups and the massless spectra depend on the choice of the complex structures on the tori, giving rise to a variety of inequivalent models. We also summarize the results for the orientifolds of the $\mathbb{Z}_{4}, \mathbb{Z}_{6}$, and $\mathbb{Z}_{6}^{\prime}$ orbifolds in four and six dimensions.
\end{abstract}

\section{KEYwORDS: 'S'Lperstring Vacua, D-branes'.}

\section{Introduction}

Orientifolds of type II string theories or, equivalently, compactifications of the type I open string theory have considerably enlarged our view on consistent vacua of string theory. The plethora of Calabi-Yau compactifications known for the heterotic string carries over to type I via the ten dimensional S-duality [1] [i]. While in ten dimensions it involves an inversion of the string coupling, this is no longer necessary in six and four dimensions. However, in the type I setting one can perturbatively study backgrounds which are non-perturbative from the heterotic viewpoint, as they they involve lower dimensional D-branes. Besides all those recent ideas arising in this context, like lower string scale unification, type I vacua still leave a variety of open questions and puzzles.

First, employing the description of type I compactifications as orientifolds of type II, one chooses an exactly solvable point in the moduli space of the Calabi-Yau manifold and performs the tadpole cancellation computation. In all known cases these exactly solvable points are

\footnotetext{
*e-mail: blumenha@physik.hu-berlin.de

†e-mail: goerlich@physik.hu-berlin.de

$\ddagger$ e-mail: koers@physik.hu-berlin.de
}

given by toroidal orbifolds or Gepner models. In general it is not clear how to deform away from these special points in moduli space. Second, at first sight surprisingly it appeared that some of the four dimensional standard orbifolds do not allow cancellation of all massless tadpoles [2]. This perturbative inconsistency has been conjectured to be cured due to non-perturbative states in [3]. However, not all cases for which perturbative inconsistencies arise have been resolved in this fashion.

Finally, the fate of the discrete NS-NS antisymmetric B-field modulus in the blown-up version and its translation to the heterotic side have not been explored in detail. Related to the presence of a B-field background is the occurance of extra multiplicities for certain open string sectors, which are required in order to achieve tadpole cancellation and anomaly freedom.

Some of these issues may possibly be tackled in a new class of orientifold models which we have presented in [4], analyzed in $[\overline{7} \mathbf{7}]$. They combine the world-sheet parity transformation with a reflection $\mathcal{R}$ of half of the internal coordinates, which can be written

\footnotetext{
${ }^{4}$ Some of the examples have been recovered in a completely different approach in [6].
} 
as a complex conjugation on any two dimensional torus. Note, that this combination still preserves half of the supersymmetry. The new features arising in this class of orientifolds are that the operation $\Omega \mathcal{R}$ does not exchange twisted sectors and that the internal B-field becomes a continuous modulus (whereas the off-diagonal entries of the metric get discretized). The extra multiplicities for certain open string sectors arise very naturally as intersection numbers of D-branes. We find solutions for any of the orbifold groups we have investigated. Even more surprisingly, we get more than just one orientifold per orbifold group, distinguished by the particular choice of the complex structures on the tori. From the phenomenological point of view the four dimensional models are of little interest, as they generically show non-chiral matter content.

In section 2 we describe the general features of $\Omega \mathcal{R}$ orientifolds. In section 3 we investigate the $\mathbb{Z}_{3}$ example as the technically most simple one in some detail. It already exhibits all conceptually new aspects of $\Omega \mathcal{R}$ orientifolds. The section 4 finally summarizes results of our computations for the $\mathbb{Z}_{4}$ and the two $\mathbb{Z}_{6}$ examples.

\section{2. $\Omega \mathcal{R}$ orientifolds}

The orientifolds with $\Omega \mathcal{R}$ projection combine the ordinary world sheet parity transformation $\Omega$ with a conjugation $\mathcal{R}$ of the complex coordinates of the compactification torus $T^{4}$ or $T^{6}$, for six- or four dimensional models respectively. The tori are taken to factorize into two dimensional tori in all cases, with coordinates given by

$$
X_{i} \equiv x_{10-2 i}+i x_{11-2 i}, \quad i=1,2,3 .
$$

The action of $\mathcal{R}$ then reads

$$
\mathcal{R}: X_{i} \mapsto \bar{X}_{i} .
$$

The complex structure of each two dimensional torus is further restricted by the requirement of the orbifold group acting crystallographically. We have chosen (up to rescaling) the $S U(3)$ or $S U(2)^{2}$ root lattices for simplicity. The relative choice of the complex structures on the various $T^{2}$ tori is of crucial importance for the gauge group and the spectra, which leads to a variety of distinct models. Since $\mathcal{R}$ can also be considered as a reflection of one half of the compact real coordinates, $\Omega \mathcal{R}$ is a symmetry of type IIA in four and of type IIB in six dimensions. This operation is then accompanied by one of the well known cyclic $\mathbb{Z}_{N}$ orbifold groups preserving $\mathcal{N}=1$ supersymmetry. We denote the groups by $\mathbb{Z}_{N}=\left\{1, \Theta, \ldots, \Theta^{N-1}\right\}$. The operation of the generator is diagonal in the complex basis:

$$
\Theta: X_{i} \mapsto \exp \left(2 \pi i v_{i}\right) X_{i}
$$

and with opposite phases on the conjugate variables. Also the fermionic coordinates are being complexified in order to diagonalize $\Theta$. The operation on the various ground states reads

$$
\begin{aligned}
\mathcal{R}:\left|s_{0}, s_{1}, s_{2}, s_{3}\right\rangle & \mapsto\left|s_{0},-s_{1},-s_{2},-s_{3}\right\rangle, \quad(2.4) \\
\Theta:\left|s_{0}, s_{1}, s_{2}, s_{3}\right\rangle & \mapsto \exp (2 \pi i \vec{v} \cdot \vec{s})\left|s_{0}, s_{1}, s_{2}, s_{3}\right\rangle .
\end{aligned}
$$

If in some twisted sector the ground state is only a spinor of a subgroup of $S O(8)$, the respective $s_{i}$ are set to zero formally. A further subtlety arises with the GSO projection which is ambiguous in the twisted closed string and the open string sectors a priori. It can be fixed by imposing the $\Omega \mathcal{R}$ symmetry of the spectrum and requiring supersymmetry. The entire orientifold group is given by $\mathbb{Z}_{N} \cup \Omega \mathcal{R} \mathbb{Z}_{N}$ and these models cannot be mapped by T-duality to standard orientifolds $\mathbb{Z}_{N} \cup \Omega \mathbb{Z}_{N}$. Instead they are dual to asymmetric orientifolds $\hat{\mathbb{Z}}_{N} \cup \Omega \hat{\mathbb{Z}}_{N}$ with standard $\Omega$ projection. The way how the T-duality acts on the various moduli of the models has been investigated in [i]".

Six dimensional $\mathbb{Z}_{N}$ orbifolds preserving $\mathcal{N}=$ 1 supersymmetry are easily defined by $v_{1}=1 / N$, $v_{2}=-1 / N$. Orbifolds featuring $\mathcal{N}=1$ supersymmetry in four dimensions have been classified in [8, 90 and we display their action on the complex basis in terms of the $v_{i}$ in table $\overline{1}_{1}^{1}$. We will discuss the $\mathbb{Z}_{3}$ example in greater detail and will only briefly state the results for the $\mathbb{Z}_{4}, \mathbb{Z}_{6}$ and $\mathbb{Z}_{6}^{\prime}$ cases. For all the other orbifold groups we do neither expect any conceptual novelties, nor will they produce more realistic models in terms of gauge groups or massless spectra, while surely demanding a large amount of tedious computation. In contrast to our results for the $\Omega \mathcal{R}$ 


$$
\begin{array}{|l|l|}
\mathbb{Z}_{3}: v=(1,1,-2) / 3 & \mathbb{Z}_{8}: v=(1,3,-4) / 8 \\
\mathbb{Z}_{4}: v=(1,1,-2) / 4 & \mathbb{Z}_{8}^{\prime}: v=(1,2,-3) / 8 \\
\mathbb{Z}_{6}: v=(1,1,-2) / 6 & \mathbb{Z}_{12}: v=(1,4,-5) / 12 \\
\mathbb{Z}_{6}^{\prime}: v=(1,2,-3) / 6 & \mathbb{Z}_{12}^{\prime}: v=(1,5,-6) / 12 \\
\mathbb{Z}_{7}: v=(1,2,-3) / 7 & \\
\hline
\end{array}
$$

Table 1: $\mathbb{Z}_{N}$ groups that preserve $\mathcal{N}=1$ in $d=4$

orientifold it has been established for the ordinary four dimensional $\mathbb{Z}_{4}$ case that there exists no solution to the tadpole condition. This is also the case for the standard $\mathbb{Z}_{8}, \mathbb{Z}_{8}^{\prime}$ and $\mathbb{Z}_{12}$ orbifolds, which we believe to have solutions as $\Omega \mathcal{R}$ orientifolds, as well.

\section{The $\mathbb{Z}_{3}$ example}

In the following we describe the computations relevant for the $\mathbb{Z}_{3}$ orientifolds in $d=4$ and $d=6$ with $\Omega \mathcal{R}$ projection in detail. The two kinds of models are technically very similar but a number of subtle distinctions need to be made with respect to the dimensionality. We shall mostly display the formulas for the six dimensional case and explain the changes in four dimensions separately. The generator $\Theta$ of the orbifold group acts diagonally by

$$
\Theta: X_{1,2} \mapsto \exp ( \pm 2 \pi i / 3) X_{1,2}
$$

in six dimensions or by

$$
\begin{aligned}
\Theta: X_{1,2} & \mapsto \exp (2 \pi i / 3) X_{1,2}, \\
\Theta: X_{3} & \mapsto \exp (-4 \pi i / 3) X_{3}
\end{aligned}
$$

in four dimensions. The torus we employ is defined by the root lattice of $S U(3)$ with basis vectors of length $\sqrt{2}$ for any of the complex directions, being depicted in Figure the fixed points of $\Theta$ by circles. The action of $\mathcal{R}$ is chosen to be a reflection of the vertical axis, leaving two inequivalent orientations of the lattice, denoted by $\mathbf{A}$ and $\mathbf{B}$. Both lattices allow a crystallographic reflection, but lead to different results for the invariant Kaluza-Klein (KK) momenta and winding (W) states as well as for the number of $\Omega \mathcal{R}$ invariant fixed points of $\Theta$.

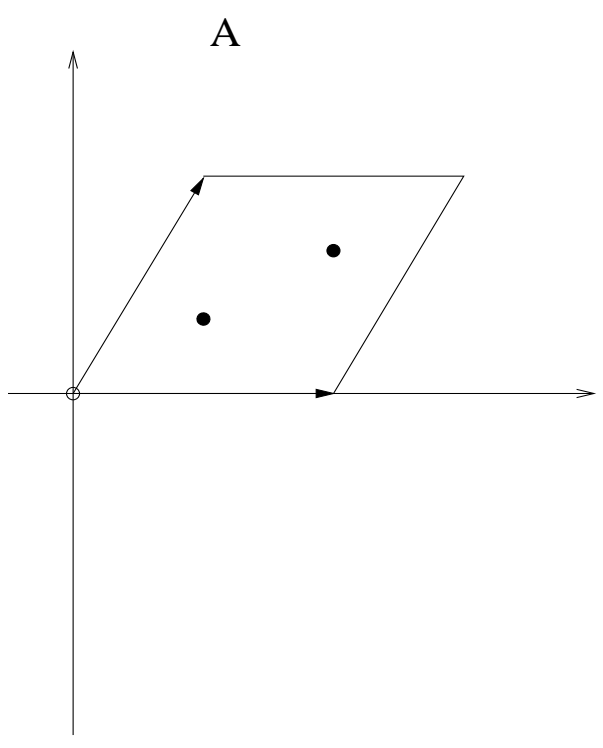

B

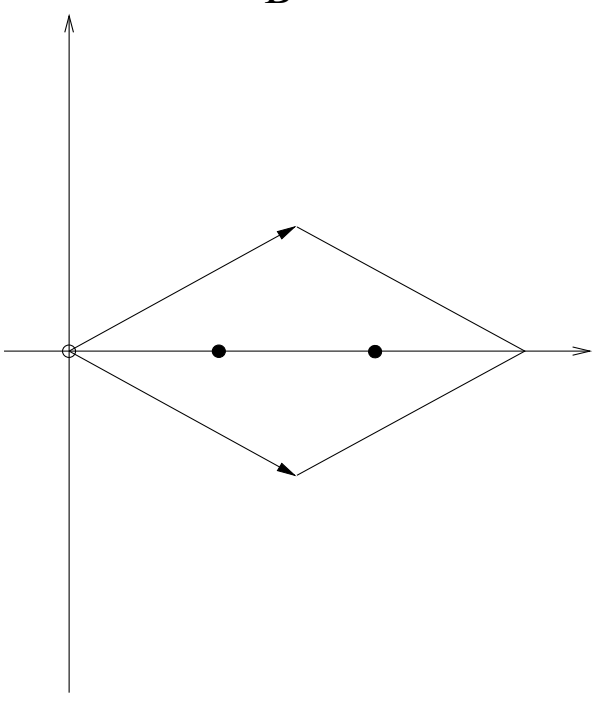

Figure 1: The $\mathbb{Z}_{3}$ lattices

\subsection{Closed string sector: The Klein bottle}

In the closed string sector we have twisted sectors for all powers of $\Theta$. The Fourier decomposition of the coordinate fields carries modings shifted by $k v_{i}$ in the $k$-twisted sector. An important feature is that $\Omega$ as well as $\mathcal{R}$ exchange twisted sectors implying that the twist fields are left invariant by the combined action. We then get the $\Omega \mathcal{R}$ invariant oscillator excitations

$$
\begin{aligned}
& (\Omega \mathcal{R}) \alpha_{n+k v_{i}} \tilde{\bar{\alpha}}_{n+k v_{i}}(\Omega \mathcal{R})^{-1}=\alpha_{n+k v_{i}} \tilde{\bar{\alpha}}_{n+k v_{i}}, \\
& (\Omega \mathcal{R}) \bar{\alpha}_{n-k v_{i}} \tilde{\alpha}_{n-k v_{i}}(\Omega \mathcal{R})^{-1}=\bar{\alpha}_{n-k v_{i}} \tilde{\alpha}_{n-k v_{i}} .
\end{aligned}
$$

The $\alpha$ denotes any kind of ladder operator in the 
$k$-twisted sector with $n \in \mathbb{Z}$ or $n \in \mathbb{Z}+1 / 2$. The states are also invariant under the action of $\Theta$ :

$$
\begin{aligned}
& \Theta \alpha_{n+k v_{i}} \tilde{\bar{\alpha}}_{n+k v_{i}} \Theta^{-1}=\alpha_{n+k v_{i}} \tilde{\bar{\alpha}}_{n+k v_{i}}, \\
& \Theta \bar{\alpha}_{n-k v_{i}} \tilde{\alpha}_{n-k v_{i}} \Theta^{-1}=\bar{\alpha}_{n-k v_{i}} \tilde{\alpha}_{n-k v_{i}} .
\end{aligned}
$$

The left- and right-moving momenta are given by

$$
\begin{aligned}
& p_{\mathrm{L}}=\frac{1}{i \sqrt{\alpha^{\prime} U_{2} T_{2}}}\left(m_{1} U-m_{2}-\bar{T}\left(n_{1}+U n_{2}\right)\right), \\
& p_{\mathrm{R}}=\frac{1}{i \sqrt{\alpha^{\prime} U_{2} T_{2}}}\left(m_{1} U-m_{2}-T\left(n_{1}+U n_{2}\right)\right),
\end{aligned}
$$

where $U$ and $T$ are the complex structure and the complexified Kähler modulus of the $T^{2}$. They are given in terms of the unit vectors spanning the lattice:

$$
\begin{aligned}
U & =\frac{e_{2}}{e_{1}}, & T & =i V, \\
e_{1}^{\mathbf{A}} & =\sqrt{2}, & e_{2}^{\mathbf{A}} & =\frac{1}{\sqrt{2}}+i \sqrt{\frac{3}{2}}, \\
e_{1}^{\mathbf{B}} & =\sqrt{2}, & e_{2}^{\mathbf{B}} & =\frac{1}{\sqrt{2}}+i \frac{1}{\sqrt{6}},
\end{aligned}
$$

where we have adopted the choice of Figure $\overline{1}_{1}^{1}$. This allows to determine the $\mathrm{KK}+\mathrm{W}$ states invariant under $\Omega \mathcal{R} \Theta^{k}$. In fact, the identity

$$
\Theta^{-1 / 2}\left(\Omega \mathcal{R} \Theta^{k}\right) \Theta^{1 / 2}=\left(\Omega \mathcal{R} \Theta^{k}\right) \Theta
$$

and the $\Theta^{-1 / 2}$ symmetry of the lattice implies that their contribution to the partition function is independent of $k$.

Using the above mentioned ingredients, the computation of the loop channel Klein bottle amplitude becomes a straightforward exercise:

$$
\begin{aligned}
\mathcal{K}= & 2^{d / 2} c \int_{0}^{\infty} \frac{d t}{t^{d / 2+1}} \operatorname{Tr}_{\mathrm{U}+\mathrm{T}}\left(\frac{\Omega \mathcal{R}}{2}\right. \\
& \left.\frac{\left(1+\Theta+\Theta^{2}\right)}{3} \frac{\left(1+(-1)^{F}\right)}{2} e^{-2 \pi t\left(L_{0}+\bar{L}_{0}\right)}\right) .
\end{aligned}
$$

We define $c \equiv V_{d} /\left(8 \pi^{2} \alpha^{\prime}\right)^{d / 2}$ and the momentum integration in the non-compact space-time has already been performed. While in the loop channel there are contributions in all twisted sectors, the relation

$$
\Omega \mathcal{R} \Theta^{k}=\Theta^{-k} \Omega \mathcal{R}
$$

implies that only untwisted closed string states propagate in the tree channel Klein bottle amplitude. More precisely, the modular transformation maps the contributions of the $k$-twisted sector to the untwisted sector with the term $\Theta^{k}$ of the orbifold projector $\left(1+\Theta+\cdots+\Theta^{N-1}\right) / N$ inserted. In order for the corresponding boundary states to be invariant under $\Theta$, it is necessary that the modular transformation produces the complete projector in the tree channel. It turns out that the latter condition is not always met automatically. We consider the completion of the projector as the guiding principle, which determines how to choose the relative orientations of the $T^{2}$ lattices, say of $\mathbf{A}$ or $\mathbf{B}$ type. Whenever this loop-tree channel equivalence is satisfied we find consistent models. In case it is not, one is actually missing states in the tree channel to complete the projector perturbatively, a circumstance that may hint towards the existence of non-perturbative states, that cure the discrepancy $\overline{1} \overline{1}$.

Luckily, for the $\mathbb{Z}_{3}$ orbifold this condition does not impose any constraints, while it does so for all the other orbifold groups. Thus we have three inequivalent choices of the lattice (their complex structures) in six dimensions:

$$
\mathbf{A A}, \mathbf{A B}, \mathbf{B B}
$$

and four in four dimensions:

$$
\text { AAA, AAB, ABB, BBB. }
$$

Another crucial input is that in the loop channel amplitude a $k$-twisted sector contribution has to be weighted by the number of fixed points of $\Theta^{k}$, which are as well invariant under $\mathcal{R}$. This factor differs for the given choices of the lattice orientations. For instance for the six dimensional AA case the loop channel Klein bottle amplitude reads

$$
\begin{aligned}
\mathcal{K}=2 & \left.c(1-1) \int_{0}^{\infty} \frac{d t}{t^{4}}\right) \frac{\vartheta\left[\begin{array}{c}
0 \\
1 / 2
\end{array}\right]^{4}}{\eta^{12}} \\
& \left(\sum_{m \in \mathbb{Z}} e^{-4 \pi t m^{2} / r^{2}}\right)^{2}\left(\sum_{n \in \mathbb{Z}} e^{-3 \pi t n^{2} r^{2}}\right)^{2}
\end{aligned}
$$




$$
\begin{gathered}
+\frac{\vartheta\left[\begin{array}{c}
0 \\
1 / 2
\end{array}\right]^{2}}{\eta^{6}} \frac{\vartheta\left[\begin{array}{l}
1 / 3 \\
1 / 2
\end{array}\right] \vartheta\left[\begin{array}{c}
-1 / 3 \\
1 / 2
\end{array}\right]}{\vartheta\left[\begin{array}{c}
-1 / 6 \\
1 / 2
\end{array}\right] \vartheta\left[\begin{array}{l}
1 / 6 \\
1 / 2
\end{array}\right]} \\
\left.+\frac{\vartheta\left[\begin{array}{c}
0 \\
1 / 2
\end{array}\right]^{2}}{\eta^{6}} \frac{\vartheta\left[\begin{array}{c}
-1 / 3 \\
1 / 2
\end{array}\right] \vartheta\left[\begin{array}{c}
1 / 3 \\
1 / 2
\end{array}\right]}{\vartheta\left[\begin{array}{l}
1 / 6 \\
1 / 2
\end{array}\right] \vartheta\left[\begin{array}{c}
-1 / 6 \\
1 / 2
\end{array}\right]}\right),
\end{gathered}
$$

the argument being $\exp (-4 \pi t)$. After a modular transformation one gets the tree channel amplitude

$$
\begin{aligned}
\tilde{\mathcal{K}}= & c \frac{32}{3}(1-1) \int_{0}^{\infty} d l\left(\frac{\vartheta\left[\begin{array}{c}
1 / 2 \\
0
\end{array}\right]}{\eta^{12}}\right. \\
& \left(\sum_{m \in \mathbb{Z}} e^{-\pi l m^{2} r^{2}}\right)^{2}\left(\sum_{n \in \mathbb{Z}} e^{-4 \pi l n^{2} /\left(3 r^{2}\right)}\right)^{2} \\
& +3 \frac{\vartheta\left[\begin{array}{c}
1 / 2 \\
0
\end{array}\right]^{2}}{\eta^{6}} \frac{\vartheta\left[\begin{array}{c}
1 / 2 \\
1 / 3
\end{array}\right] \vartheta\left[\begin{array}{c}
1 / 2 \\
-1 / 3
\end{array}\right]}{\vartheta\left[\begin{array}{c}
1 / 2 \\
-1 / 6
\end{array}\right] \vartheta\left[\begin{array}{c}
1 / 2 \\
1 / 6
\end{array}\right]} \\
& \left.+3 \frac{\vartheta\left[\begin{array}{c}
1 / 2 \\
0
\end{array}\right]^{2}}{\eta^{6}} \frac{\vartheta\left[\begin{array}{c}
1 / 2 \\
-1 / 3
\end{array}\right] \vartheta\left[\begin{array}{c}
1 / 2 \\
1 / 3
\end{array}\right]}{\vartheta\left[\begin{array}{c}
1 / 2 \\
1 / 6
\end{array}\right] \vartheta\left[\begin{array}{c}
1 / 2 \\
-1 / 6
\end{array}\right]}\right)
\end{aligned}
$$

The relative factors $(2 \sin (\pi / 3))^{2}=3$ between the different contributions to the tree channel amplitude come out just as expected to complete the projector. Switching to the $\mathbf{B}$ lattice in any complex direction results in an overall factor of 3 , while changing the lattice contributions and the number of $\mathcal{R}$ invariant fixed points of $\Theta$.

For computing the massless closed string spectra one also needs to distinguish the various lattice choices very carefully. The untwisted $\Theta$ invariant states simply have to be symmetrized and antisymmetrized under $\Omega \mathcal{R}$ in the NSNS and the $\mathrm{RR}$ sector respectively. This contribution to the spectrum is generic and together with the spectrum of the twisted sectors has been summarized in table dilaton multiplets. The spectrum coming from twisted sectors differs for fixed points invariant under $\Omega \mathcal{R}$ and for those fixed points, that form pairs under $\Omega \mathcal{R}$. The former fixed points contribute two hypermultiplets in six and a chiral multiplet in four dimensions. The latter fixed points contribute two hypermultiplets in addition to two tensor multiplets in six dimensions and

\begin{tabular}{|c|c|c|c|}
\hline Dim. & Model & untwisted & $\begin{array}{c}\Theta+\Theta^{-1} \\
\text { twisted }\end{array}$ \\
\hline \multirow{3}{*}{$d=6$} & AA & $3 \mathrm{H}$ & $10 \mathrm{H}+8 \mathrm{~T}$ \\
& AB & $3 \mathrm{H}$ & $12 \mathrm{H}+6 \mathrm{~T}$ \\
& BB & $3 \mathrm{H}$ & $18 \mathrm{H}$ \\
\hline \multirow{5}{*}{$d=4$} & AAA & $9 \mathrm{C}$ & $14 \mathrm{C}+13 \mathrm{~V}$ \\
& AAB & $9 \mathrm{C}$ & $15 \mathrm{C}+12 \mathrm{~V}$ \\
& ABB & $9 \mathrm{C}$ & $18 \mathrm{C}+9 \mathrm{~V}$ \\
& BBB & $9 \mathrm{C}$ & $27 \mathrm{C}$ \\
\hline
\end{tabular}

Table 2: Closed string spectra

a chiral plus a vector multiplet in four dimensions. The sum of the neutral multiplets from the closed string sector finally matches the sum of the Hodge numbers $h^{1,1}+h^{1,2}$ in all cases.

\subsection{Open string sector: The annulus and the Möbius strip}

To cancel the tadpoles from the Klein bottle one has to introduce $\mathrm{D}(d / 2+5)$-branes into the background, which intersect each other on the tori at non-trivial angles. They are extended in one dimension on each two dimensional torus and need to be located in a $\Theta$ and $\mathcal{R}$ invariant way. Recalling that open string coordinates with boundary conditions

$$
\Re\left(\frac{\partial}{\partial \sigma} X^{i}\right)=0, \quad \Im\left(\frac{\partial}{\partial \tau} X^{i}\right)=0
$$

at $\sigma=0$ and

$$
\Re\left(e^{\pi i w_{i}} \frac{\partial}{\partial \sigma} X^{i}\right)=0, \quad \Im\left(e^{\pi i w_{i}} \frac{\partial}{\partial \tau} X^{i}\right)=0
$$

at $\sigma=\pi$ have a Fourier decomposition with modings shifted by $w_{i}$, we are led to consider arrays of 3 kinds of D-branes at relative angle $\pi / 3$. On each torus $T^{2}$ one of them is located in the fixed plane of the reflection $\mathcal{R}$ and the remaining ones are obtained by successively applying the rotation $\Theta^{1 / 2}$. Such a configuration is shown in Figure $\overline{2}_{\mathbf{r}}$ The type of lattice is being distinguished, as the number of intersection points of any two kinds of branes differs. One now needs to compute the annulus

$$
\begin{gathered}
\mathcal{A}=c \int_{0}^{\infty} \frac{d t}{t^{d / 2+1}} \operatorname{Tr}_{\text {open }}\left(\frac{1}{2} \frac{1+\Theta+\Theta^{2}}{3}\right. \\
\left.\frac{1+(-1)^{F}}{2} e^{-2 \pi t L_{0}}\right)
\end{gathered}
$$



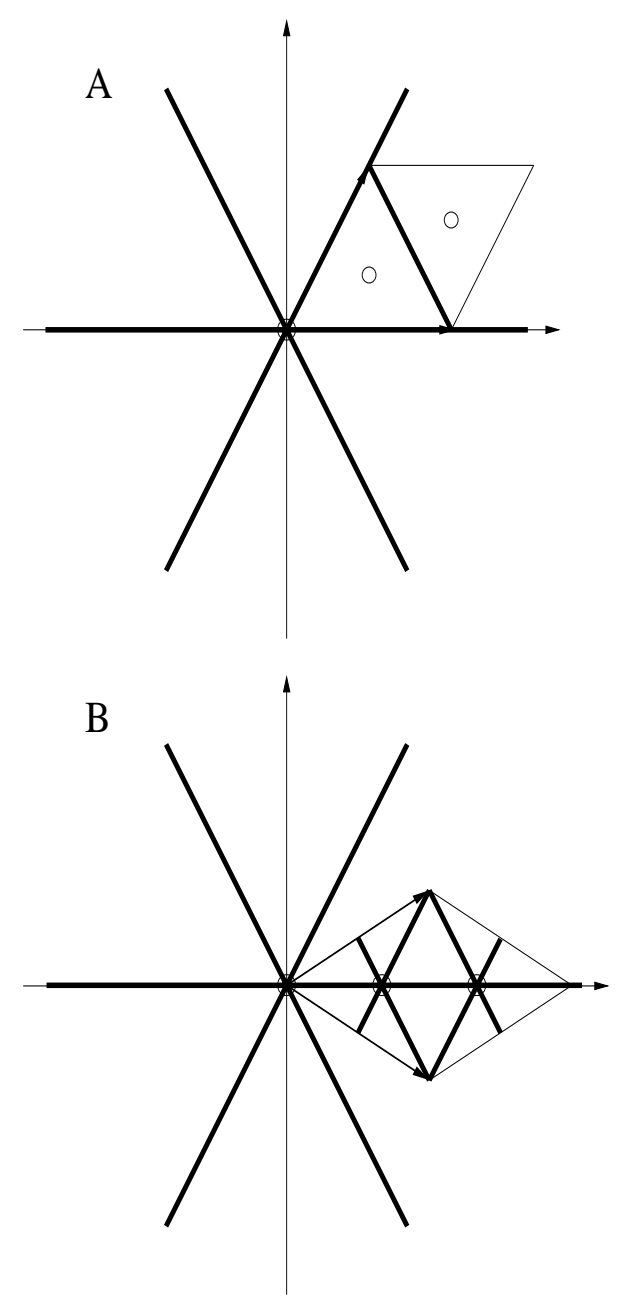

Figure 2: D-branes on the $S U(3)$ root lattice

and Möbius strip amplitudes

$$
\begin{aligned}
\mathcal{M}=c & \int_{0}^{\infty} \frac{d t}{t^{d / 2+1}} \operatorname{Tr}_{\text {open }}\left(\frac{\Omega \mathcal{R}}{2} \frac{1+\Theta+\Theta^{2}}{3}\right. \\
& \left.\frac{1+(-1)^{F}}{2} e^{-2 \pi t L_{0}}\right)
\end{aligned}
$$

There are only non-vanishing contributions in the traces, if the operator in the trace leaves the two D-branes $\left(\mathrm{D}_{i}, \mathrm{D}_{i+n}\right)$, the string stretches between, and its orientation invariant. If the moding of the fields in the $\left(\mathrm{D}_{i}, \mathrm{D}_{i+n}\right)$ open string sector is identical to the moding of the fields in the $\Theta^{k}$ twisted closed string sector, we call such a sector the " $\Theta^{k}$ twisted" open string sector.

In order to compute the zero-mode contributions to the $\left(\mathrm{D}_{i}, \mathrm{D}_{i}\right)$ amplitudes, one needs to inspect invariant $\mathrm{KK}+\mathrm{W}$ states. The result is sensitive to the type of lattice. The additional requirement, that the Möbius strip contributions have to be $\Omega \mathcal{R}$ invariant, induces a doubling of winding numbers as compared to the annulus. A very interesting point to notice is an extra multiplicity of some twisted open string sectors. As can be seen in Figure 2 , the different kinds of branes intersect only in the origin of the $\mathbf{A}$ type unit cell, while they intersect in all three fixed points of $\Theta$ in the $\mathbf{B}$ type unit cell. Thus the corresponding twisted sectors have to be weighted with extra multiplicities, leading to the same extra multiplicity for the number of states at each mass level. This turns out to be necessary to obtain anomaly free massless spectra. Note, that this issue has been related via T-duality to extra factors for open string sectors in ordinary $\Omega$ ori-

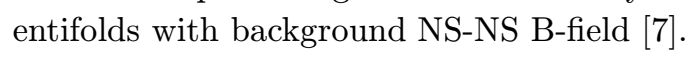

We present the tree channel annulus and Möbius strip amplitude in six dimensions for the AA choice of the lattice. Switching to a $\mathbf{B}$ type lattice results in an overall factor of 3 for all amplitudes and has an effect on the massless spectrum. The annulus reads

$$
\begin{aligned}
\tilde{\mathcal{A}}= & c \frac{\mathrm{M}^{2}}{6}(1-1) \int_{0}^{\infty} d l\left(\frac{\vartheta\left[\begin{array}{c}
1 / 2 \\
0
\end{array}\right]}{\eta^{12}}\right. \\
& \left(\sum_{m \in \mathbb{Z}} e^{-\pi l m^{2} r^{2}}\right)^{2}\left(\sum_{n \in \mathbb{Z}} e^{-4 \pi l n^{2} /\left(3 r^{2}\right)}\right)^{2} \\
& +3 \frac{\vartheta\left[\begin{array}{c}
1 / 2 \\
0
\end{array}\right]^{2}}{\eta^{6}} \frac{\vartheta\left[\begin{array}{c}
1 / 2 \\
1 / 3
\end{array}\right] \vartheta\left[\begin{array}{c}
1 / 2 \\
-1 / 3
\end{array}\right]}{\vartheta\left[\begin{array}{c}
1 / 2 \\
-1 / 6
\end{array}\right] \vartheta\left[\begin{array}{c}
1 / 2 \\
1 / 6
\end{array}\right]} \\
& \left.+3 \frac{\vartheta\left[\begin{array}{c}
1 / 2 \\
0
\end{array}\right]^{2}}{\eta^{6}} \frac{\vartheta\left[\begin{array}{c}
1 / 2 \\
-1 / 3
\end{array}\right] \vartheta\left[\begin{array}{c}
1 / 2 \\
1 / 3
\end{array}\right]}{\vartheta\left[\begin{array}{c}
1 / 2 \\
1 / 6
\end{array}\right] \vartheta\left[\begin{array}{c}
1 / 2 \\
-1 / 6
\end{array}\right]}\right)
\end{aligned}
$$

with argument $\exp (-4 \pi l)$ and the Möbius strip gives

$$
\begin{aligned}
\tilde{\mathcal{M}}= & -c \frac{8 \mathrm{M}}{3}(1-1) \int_{0}^{\infty} d l\left(\frac{\vartheta\left[\begin{array}{c}
1 / 2 \\
0
\end{array}\right]^{4} \vartheta\left[\begin{array}{c}
0 \\
1 / 2
\end{array}\right]^{4}}{\eta^{12} \vartheta\left[\begin{array}{l}
0 \\
0
\end{array}\right]^{4}}\right. \\
& \left(\sum_{m \in \mathbb{Z}} e^{-4 \pi l m^{2} r^{2}}\right)^{2}\left(\sum_{n \in \mathbb{Z}} e^{-4 \pi l n^{2} /\left(3 r^{2}\right)}\right)^{2}
\end{aligned}
$$




$$
\begin{gathered}
+3 \frac{\vartheta\left[\begin{array}{c}
1 / 2 \\
0
\end{array}\right]^{2} \vartheta\left[\begin{array}{c}
0 \\
1 / 2
\end{array}\right]^{2}}{\eta^{6} \vartheta\left[\begin{array}{l}
0 \\
0
\end{array}\right]^{2}} \\
\left(\frac{\vartheta\left[\begin{array}{c}
1 / 2 \\
1 / 3
\end{array}\right] \vartheta\left[\begin{array}{c}
0 \\
-1 / 6
\end{array}\right] \vartheta\left[\begin{array}{c}
1 / 2 \\
-1 / 3
\end{array}\right] \vartheta\left[\begin{array}{c}
0 \\
1 / 6
\end{array}\right]}{\vartheta\left[\begin{array}{c}
0 \\
1 / 3
\end{array}\right] \vartheta\left[\begin{array}{c}
1 / 2 \\
-1 / 6
\end{array}\right] \vartheta\left[\begin{array}{c}
0 \\
-1 / 3
\end{array}\right] \vartheta\left[\begin{array}{c}
1 / 2 \\
1 / 6
\end{array}\right]}\right. \\
\left.\left.+\frac{\vartheta\left[\begin{array}{c}
1 / 2 \\
-1 / 3
\end{array}\right] \vartheta\left[\begin{array}{c}
0 \\
1 / 6
\end{array}\right] \vartheta\left[\begin{array}{c}
1 / 2 \\
1 / 3
\end{array}\right] \vartheta\left[\begin{array}{c}
0 \\
-1 / 6
\end{array}\right]}{\vartheta\left[\begin{array}{c}
0 \\
-1 / 3
\end{array}\right] \vartheta\left[\begin{array}{c}
1 / 2 \\
1 / 6
\end{array}\right] \vartheta\left[\begin{array}{c}
0 \\
1 / 3
\end{array}\right] \vartheta\left[\begin{array}{c}
1 / 2 \\
-1 / 6
\end{array}\right]}\right)\right) .
\end{gathered}
$$

with argument $\exp (-8 \pi l)$. One obtains only a single overall tadpole cancellation condition, which reads

$$
\frac{1}{6}\left(\mathrm{M}^{2}-16 \mathrm{M}+64\right)=\frac{1}{6}(\mathrm{M}-8)^{2}=0 .
$$

It implies the presence of $\mathrm{M}=8 \mathrm{D} 7$-branes of each kind. The analogous condition in four dimensions requires $\mathrm{M}=4 \mathrm{D} 6$-branes. The tadpoles are related to contributions of 8- and 7forms respectively. Thus, we get an $S O(8)$ gauge group in six dimensions and $S O(4)$ in four. The rank reduction with respect to the $S O(32)$ in ten dimensions can be related to the rank reduction in ordinary $\Omega$ orientifolds with background Bfield via T-duality. As was pointed out in [i it is possible via discrete Wilson-lines to get the gauge groups $S p(8)$ and $S p(4)$, respectively.

The massless spectrum in the untwisted open string sector is again generic. In six dimensions there is a hypermultiplet, in four dimensions three chiral multiplets in addition to the gauge vectors, all in the antisymmetric representation. In the twisted sectors one needs to count the extra multiplicity of states deriving from the number of intersection points, each giving rise to a hypermultiplet in the antisymmetric in six and a chiral multiplet in the symmetric representation in four dimensions. The open string spectra are collected in table $\underline{\beta_{1}^{1}}$. All the six dimensional models satisfy the cancellation condition of the irreducible anomaly

$$
n_{\mathrm{H}}-n_{\mathrm{V}}+29 n_{\mathrm{T}}=273
$$

while the four dimensional models are free of any gauge anomaly anyway.

\begin{tabular}{|c|c|c|c|}
\hline Dim. & Model & $\left(\mathrm{D}_{i}, \mathrm{D}_{i}\right)$ & $\left(\mathrm{D}_{i}, \mathrm{D}_{i+1}\right)$ \\
\hline \multirow{4}{*}{$d=6$} & AA & $1 \mathrm{H}$ in $(\mathbf{2 8})$ & $1 \mathrm{H}$ in $(\mathbf{2 8})$ \\
& $\mathbf{A B}$ & $1 \mathrm{H}$ in $(\mathbf{2 8})$ & $3 \mathrm{H}$ in $(\mathbf{2 8})$ \\
& AA & $1 \mathrm{H}$ in $(\mathbf{2 8})$ & $9 \mathrm{H}$ in $(\mathbf{2 8})$ \\
\hline \multirow{5}{*}{$d=4$} & AAA & $3 \mathrm{C}$ in $(\mathbf{6})$ & $1 \mathrm{C}$ in $(\mathbf{1 0})$ \\
& AAB & $3 \mathrm{C}$ in $(\mathbf{6})$ & $3 \mathrm{C}$ in $(\mathbf{1 0})$ \\
& ABB & $3 \mathrm{C}$ in $(\mathbf{6})$ & $9 \mathrm{C}$ in $(\mathbf{1 0})$ \\
& BBB & $3 \mathrm{C}$ in $(\mathbf{6})$ & $27 \mathrm{C}$ in $(\mathbf{1 0})$ \\
\hline
\end{tabular}

Table 3: Open string spectra

\section{Results for $\mathbb{Z}_{4}, \mathbb{Z}_{6}$ and $\mathbb{Z}_{6}^{\prime}$}

In this section we shall only briefly explain some further properties of the other models which we have computed so far. The lattice and brane configurations, the explicit formulas for all the amplitudes to be calculated as well as all their

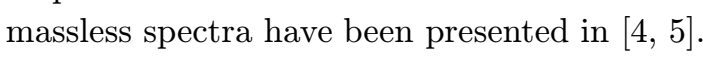

The world-sheet consistency condition is more restrictive than for the $\mathbb{Z}_{3}$, where all combinations of lattices were allowed. For all other examples we find that the relative orientation is fixed between two of the complex tori, while, in four dimensions, the third can be chosen freely of A or B type. With this freedom we obtain another set of eight inequivalent models from the three orbifold groups.

In the open string sector one again has to introduce sets of branes at $\pi v_{i}$ relative angles, where their respective number has to be determined by the tadpole cancellation conditions. Since there always exist two types of branes which are not mapped upon each other by the orbifold, the gauge group will have a product structure with two identical factors. The spectra, however, need not be symmetric under exchanging the two factors. As all the orbifold groups contain an element $\Theta^{N / 2}$ of order two, there is also a contribution to the $\mathbb{Z}_{2}$ twisted sector in the tree channel. This generically imposes the second tadpole cancellation condition

$$
\operatorname{tr}\left(\gamma_{N / 2}^{(i)}\right)=0
$$

for the action of $\Theta^{N / 2}$ on the Chan-Paton factors of each type $i$ of branes. The two conditions together then exactly reproduce the situation of the standard six dimensional $\mathbb{Z}_{2}$ orientifold discussed 
by Gimon and Polchinski in [1] i in. Their solution implies that the orthogonal $S O(\mathrm{M})$ gauge group is broken to its unitary $U(\mathrm{M} / 2)$ subgroup. For instance, in four dimensions for the $\mathbb{Z}_{4}$ orbifold we find two possible solutions $U(8)^{2}$ and $U(4)^{2}$, while for the various $\mathbb{Z}_{6}$ models we always get $U(2)^{2}$. This is in accord with the rank reduction expected from T-duality.

The determination of the massless spectra is in general more involved than for the $\mathbb{Z}_{3}$. In the twisted sectors of the closed string spectrum one has to keep track of the transformation properties of the various fixed points under the orbifold group generator $\Theta$ and $\Omega \mathcal{R}$ in order to find the correct symmetrization prescription. Also one needs to distinguish with respect to the action of $\Theta^{N / 2}$. In the open string spectrum the same is true for the actions on the intersection points, which do not simply provide all the same states anymore. In four dimensions there are certain subtle phase factors to be regarded, which sometimes require extra signs in the loop channel amplitude in order to complete the tree channel projector. Finally, for the most complicated case, the $\mathbb{Z}_{6}^{\prime}$, one even needs to distinguish different types of branes with different effective gauge theories on them. The results display spectra which are anomaly free in six dimensions and non-chiral in four.

\section{Acknowledgments}

We would like to thank C. Angelantonj, who participated in closely related work, A. Kumar, who was also involved in an early stage, as well as M. Gaberdiel and D. Lüst for encouraging discussions and helpful remarks.

\section{References}

[1] J. Polchinski and E. Witten, Evidence for heterotic - type I string duality, Nucl. Phys. B460 (1996) 525-540, [hep-th/9510169i].

[2] G. Aldazabal, A. Font, L. E. Ibanez, and G. Violero, $D=4, N=1$, type $I I B$ orientifolds, Nucl. Phys. B536 (1998) 29, hep-th/9804026".
[3] Z. Kakushadze, G. Shiu, and S. H. H. Tye, Type IIB orientifolds, F-theory, type I strings on orbifolds and type I heterotic duality, Nucl. Phys. B533 (1998) 25, [hep-th/9804092].

[4] R. Blumenhagen, L. Görlich, and B. Kors, Supersymmetric orientifolds in $6 D$ with $D$-branes at angles, hep-th/9908130;

[5] R. Blumenhagen, L. Görlich, and B. Kors, Supersymmetric $4 D$ orientifolds of type IIA with D6-branes at angles, hep-th/9912204.

[6] G. Pradisi, Type I vacua from diagonal Z(3)-orbifolds, hep-th/9912218.

[7] C. Angelantonj and R. Blumenhagen, Discrete deformations in type I vacua, hep-th/9911190!

[8] L. Dixon, J. A. Harvey, C. Vafa, and E. Witten, Strings on orbifolds, Nucl. Phys. B261 (1985) 678-686.

[9] L. Dixon, J. A. Harvey, C. Vafa, and E. Witten, Strings on orbifolds. 2, Nucl. Phys. B274 (1986) 285-314.

[10] Z. Kakushadze, Non-perturbative orientifolds, Phys. Lett. B455 (1999) 120, hep-th/99040077.

[11] E. G. Gimon and J. Polchinski, Consistency conditions for orientifolds and D-manifolds, Phys. Rev. D54_(1996) 1667-1676, hep-th/9601038i. 